\title{
Shock-Wave Equation-of-State Measurements in Fused Silica up to 1600 GPa
}

\section{Introduction}

Silica is the single most-abundant compound in the earth's mantle and crust and an end member of the $\mathrm{MgO}-\mathrm{FeO}-\mathrm{SiO}_{2}$ system that forms those structures. ${ }^{1-3}$ Its behavior at high pressure is fundamental for studies of interest to geophysics, tectonophysics, and the formation of exoplanets. ${ }^{4}$ In both amorphous and crystalline forms, it is a prototype for studying materials at extreme conditions using both static $\mathrm{c}^{5-7}$ and dynamic $^{8-10}$ compression experiments. The variety of stable polymorphs that exist at ambient pressure makes it possible to cover a wide region of phase space with single-shock techniques. This made it possible to approximate the Grüneisen parameter for solid silica. ${ }^{11-13}$

Until recently, most high-pressure data for silica were for pressures below $200 \mathrm{GPa}$, exploring melting and different phase changes in the structure. The development of drivers and techniques at higher pressures has made it possible to study fluid silica produced by TPa shocks using various polymorphs of silica. Understanding material behavior at these extreme conditions is of interest for planetary astrophysics, equation-of-state (EOS) research, and inertial confinement fusion. The principal Hugoniot $^{14-16}$ and release isentrope ${ }^{17-19}$ of $\alpha$-quartz was measured in the fluid regime (>100 GPa). Research has also been conducted in fused silica, ${ }^{16,20-23}$ stishovite, ${ }^{21}$ and low-density silica foams. ${ }^{24-26}$ This research has enabled one to understand the behavior of materials at the core-mantle boundary on the earth's pressure-temperature conditions where silica would be fluid. ${ }^{27}$ At higher pressures ( $\left.>300 \mathrm{GPa}\right)$, the compressed-silica EOS describes the behavior of material at the core-mantle boundary in super-Earths and other giant exoplanets. ${ }^{21}$ For further increases in pressure along the Hugoniot, silica transitions from a bonded molecular liquid to a dissociated atomic fluid. ${ }^{16}$ Alpha-quartz is frequently used as an impedance-matching standard for experiments above $300 \mathrm{GPa}$ (Refs. 17 and 19), allowing one to study other materials at high pressure. These data on fused silica provide added information about silica in this regime.

This article presents results of precision EOS measurements of the fused-silica Hugoniot from 200 to $1600 \mathrm{GPa}$ using the impedance-matching technique. Previous work measured the fused-silica Hugoniot above the melt curve from 200 to $900 \mathrm{GPa}$ (Refs. 20 and 22). This work extends measurements of the fused-silica Hugoniot well into the dissociated regime and provides lower-pressure data that agree with that of Qi et al. ${ }^{22}$ The latter is significant in that the results of laser-driven experiments using $\alpha$-quartz as an impedance-matching standard agree well with those from direct-impact measurements using aluminum flyer plates driven by magnetic-field acceleration. Additionally, this agreement increases confidence in the release model recently developed for silica (quartz) as a standard. ${ }^{19}$

\section{Method}

The Rankine-Hugoniot relations relate the conditions behind a shock discontinuity to those in front of it. ${ }^{28}$ The relations for conservation of mass and momentum give

$$
\begin{gathered}
\frac{\rho_{0}}{\rho}=1-\frac{\left(u_{\mathrm{p}}\right)}{U_{\mathrm{s}}}, \\
P=\rho_{0} U_{\mathrm{s}} u_{\mathrm{p}},
\end{gathered}
$$

where $\rho$ is the density, $\rho_{0}$ is the initial (unshocked) density, $u_{\mathrm{p}}$ is the particle velocity, $U_{\mathrm{s}}$ is the shock velocity, and $P$ is the pressure. If $P_{0}$ and $\rho_{0}$ are known, measurement of two quantities (typically $U_{\mathrm{s}}$ and $u_{\mathrm{p}}$ ) will close these equations and provide an EOS point on the material's Hugoniot.

In this work, the Hugoniot of fused silica was determined by impedance matching (IM) to an $\alpha$-quartz standard. The IM method relies on the Hugoniot and release curve of a standard to infer the sample's particle velocity from measurements of shock velocity in the standard and the sample. Quartz has recently been established as the preferred standard for EOS experiments because of its transparency at ambient conditions and high shock reflectivity. ${ }^{17,19}$ This technique used the quartz Hugoniot and release curves derived by Knudson and Desjarlais. ${ }^{15,19}$ 
These experiments comprise ten older, previously unpublished experiments on the OMEGA laser (shots 55499 to 55508 and 64348$)^{29}$ and 18 recent experiments using the OMEGA EP laser. ${ }^{30}$ Both of these frequency-tripled Nd:glass lasers operate at a wavelength of $351 \mathrm{~nm}$. The shock pressures in these experiments were generated using laser pulses ranging from 2- to 6-ns duration, with intensities ranging from $\sim 0.2$ to $1.7 \times 10^{14} \mathrm{~W} / \mathrm{cm}^{2}$. The laser spots were all smoothed using distributed phase plates ${ }^{31}$ to achieve shocks with planar regions of either 750 or $1100 \mu \mathrm{m}$ in diameter.

The targets consisted of 3-mm $\times 3$-mm flat, $z$-cut, $\alpha$-quartz baseplates with a nominal thickness of either 50 or $75 \mu \mathrm{m}$. A $15-\mu \mathrm{m}$-thick layer of parylene $(\mathrm{CH})$ was deposited on the front of the target as a low- $Z$ ablator that enhanced the ablation pressure and reduced the production of hard $x$ rays. The laser plasmas produced in this case had temperatures of $\sim 2 \mathrm{keV}$ (Ref. 32) such that the attenuation length of $x$ rays in the quartz was $<5 \mu \mathrm{m}$ (Ref. 33). This limits the preheating of the quartz baseplate to regions far from the point where IM is performed. Fused-silica and $\alpha$-quartz samples were glued, adjacent to each other, to the back of the baseplate. These were affixed to the baseplate using an ultralow viscosity, UV-cured epoxy that produced glue layers $<3 \mu \mathrm{m}$ thick. A sketch of the target assembly is shown in Fig. 143.19(a). The fused-silica and quartz samples had initial densities of $2.20 \mathrm{~g} / \mathrm{cm}^{3}$ and $2.65 \mathrm{~g} / \mathrm{cm}^{3}$, respectively. The refractive indices at $532 \mathrm{~nm}$ were 1.461 for the fused silica and 1.547 for the quartz.

Shock velocities were measured using a line-imaging velocity interferometer system for any reflector (VISAR) ${ }^{34-37}$ that measures the phase change caused by Doppler-shifted light reflecting off a moving surface. At pressures above $150 \mathrm{GPa}$, both the fused silica and quartz melt and form reflective shock fronts. ${ }^{16}$ The materials are both initially transparent so VISAR measures the velocity of the shock front within the material. Antireflective coatings were applied to the back surface of both samples to eliminate ghost reflections from that surface. To resolve $2 \pi$ ambiguities, two VISAR's with different velocity sensitivities were used. These sensitivities were $3.194 \mathrm{~km} / \mathrm{s} /$ fringe and $4.375 \mathrm{~km} / \mathrm{s} /$ fringe for the quartz and $3.389 \mathrm{~km} / \mathrm{s} /$ fringe and $4.642 \mathrm{~km} / \mathrm{s} /$ fringe for the fused silica. The VISAR images provided phase shifts resulting from changes in velocity that were analyzed using the Fourier transform method. ${ }^{37}$ The error in determining the phase was estimated as $\sim 3 \%$ of a fringe, resulting in velocity measurements with $<1 \%$ precision because of multiple fringe jumps produced by the shock. The VISAR system used a frequencydoubled Nd:YAG laser operating at $532 \mathrm{~nm}$, and the two streak cameras used 9- and 15-ns sweep durations. Figures 143.19(b) and 143.19(c) provide an example of the raw data and the extracted velocity profile.

The experimental observables were the shock velocity in the quartz and fused-silica samples at the point at which the shock transited the quartz-fused silica interface. The shock velocities in the quartz and fused silica were measured immediately before and after the interface, respectively. The quartz witness (adjacent to the fused-silica sample) monitored the velocity across the glue layer and the planarity of the shock. The initial shock velocity in the fused silica was fit over 300 ps and linearly extrapolated back to the quartz/glue interface to (a)

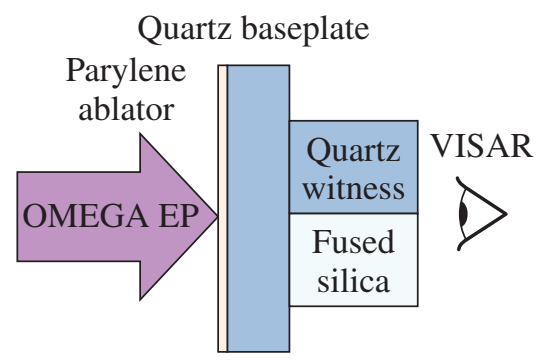

E24319JR (b)

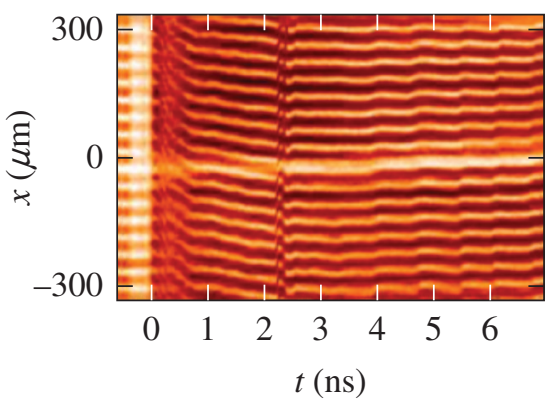

(c)

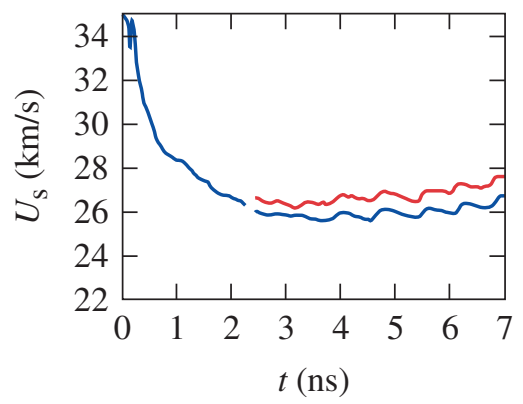

Figure 143.19

(a) Target schematic. (b) Raw VISAR data for shot 17862. Target orientation is the same as in (a). (c) Extracted velocity for shot 17862 showing quartz (blue) and fused silica (red). VISAR: velocity interferometer system for any reflector. 
determine the velocity at the location of the interface, assuming no glue layer existed. ${ }^{38}$

Errors were calculated using the Monte Carlo method, which generates normally distributed random variables based on the mean and standard deviation of an observed quantity. For these shock velocities, this resulted in an observation error $<1 \%$ from VISAR. The initial density was assumed to have negligible error, and the errors in the quartz Hugoniot and release curve were determined from the covariance matrices for the cubic Hugoniot and effective Grüneisen parameter from Ref. 19.

\section{Results/Discussion}

A total of 28 impedance-matching points were used to determine the Hugoniot of fused silica; they ranged in pressure from $260 \mathrm{GPa}$ up to $1570 \mathrm{GPa}$. The results are given in Table 143.I. The shock speed's dependence on particle velocity $\left(U_{\mathrm{s}}-u_{\mathrm{p}}\right.$ curve), shown in Fig. 143.20(a), was fit to a cubic polynomial (red dashed line), and the exponential form $U_{\mathrm{s}}=a+b u_{\mathrm{p}}-c u_{\mathrm{p}} e^{-d u_{\mathrm{p}}}$ (green line). These are shown as well as the exponential fit from Qi et al. ${ }^{22}$ (black dashed-dotted line). The error bars on the data are smaller than the points in the graph. The cubic and exponential fits were made using

Table 143.I: Shock parameters for fused-silica determined from impedance matching to quartz standard.

\begin{tabular}{|c|c|c|c|c|c|}
\hline Shot & $U_{\mathrm{s}}^{Q}(\mathrm{~km} / \mathrm{s})$ & $U_{\mathrm{s}}^{\mathrm{FS}}(\mathrm{km} / \mathrm{s})$ & $u_{\mathrm{p}}^{\mathrm{FS}}(\mathrm{km} / \mathrm{s})$ & $P^{\mathrm{FS}}(\mathrm{GPa})$ & $\rho^{\mathrm{FS}}\left(\mathrm{g} / \mathrm{cm}^{3}\right)$ \\
\hline 55499 & $21.58 \pm 0.05$ & $21.83 \pm 0.06$ & $13.85 \pm 0.05$ & $665.5 \pm 2.8$ & $6.02 \pm 0.05$ \\
\hline 55500 & $26.33 \pm 0.05$ & $26.92 \pm 0.06$ & $17.85 \pm 0.06$ & $1057.8 \pm 3.7$ & $6.53 \pm 0.05$ \\
\hline 55501 & $30.18 \pm 0.05$ & $30.88 \pm 0.06$ & $21.21 \pm 0.08$ & $1441.4 \pm 6.0$ & $7.03 \pm 0.07$ \\
\hline 55502 & $31.32 \pm 0.05$ & $32.11 \pm 0.06$ & $22.19 \pm 0.10$ & $1568.6 \pm 7.6$ & $7.13 \pm 0.08$ \\
\hline 55503 & $27.00 \pm 0.05$ & $27.30 \pm 0.06$ & $18.50 \pm 0.06$ & $1111.5 \pm 3.8$ & $6.83 \pm 0.06$ \\
\hline 55505 & $23.51 \pm 0.05$ & $24.02 \pm 0.06$ & $15.57 \pm 0.06$ & $823.1 \pm 3.2$ & $6.26 \pm 0.05$ \\
\hline 55506 & $18.27 \pm 0.05$ & $18.37 \pm 0.06$ & $11.24 \pm 0.05$ & $454.3 \pm 2.2$ & $5.67 \pm 0.05$ \\
\hline 55507 & $17.25 \pm 0.05$ & $17.26 \pm 0.06$ & $10.39 \pm 0.05$ & $394.7 \pm 2.1$ & $5.53 \pm 0.05$ \\
\hline 55508 & $14.43 \pm 0.14$ & $14.44 \pm 0.06$ & $8.40 \pm 0.13$ & $267.1 \pm 4.1$ & $5.26 \pm 0.12$ \\
\hline 64348 & $21.81 \pm 0.10$ & $22.16 \pm .017$ & $14.02 \pm 0.11$ & $683.8 \pm 6.2$ & $5.99 \pm 0.13$ \\
\hline 18752 & $23.23 \pm 0.10$ & $23.51 \pm 0.13$ & $15.22 \pm 0.11$ & $787.5 \pm 6.2$ & $6.24 \pm 0.11$ \\
\hline 18754 & $21.48 \pm 0.10$ & $21.59 \pm 0.10$ & $13.79 \pm 0.10$ & $655.4 \pm 5.3$ & $6.09 \pm 0.10$ \\
\hline 18755 & $24.60 \pm 0.10$ & $24.89 \pm 0.10$ & $16.41 \pm 0.11$ & $899.2 \pm 6.3$ & $6.46 \pm 0.10$ \\
\hline 18757 & $22.18 \pm 0.10$ & $22.33 \pm 0.17$ & $14.37 \pm 0.11$ & $706.5 \pm 6.3$ & $6.18 \pm 0.14$ \\
\hline 18758 & $25.43 \pm 0.10$ & $25.72 \pm 0.10$ & $17.13 \pm 0.11$ & $969.9 \pm 6.6$ & $6.59 \pm 0.11$ \\
\hline 18760 & $26.24 \pm 0.10$ & $26.62 \pm 0.10$ & $17.82 \pm 0.11$ & $1043.9 \pm 6.9$ & $6.66 \pm 0.11$ \\
\hline 18761 & $23.47 \pm 0.10$ & $23.54 \pm 0.14$ & $15.49 \pm 0.11$ & $802.6 \pm 6.4$ & $6.44 \pm 0.13$ \\
\hline 18762 & $26.34 \pm 0.10$ & $26.49 \pm 0.10$ & $17.95 \pm 0.11$ & $1046.7 \pm 7.0$ & $6.83 \pm 0.11$ \\
\hline 18763 & $24.39 \pm 0.10$ & $24.32 \pm 0.10$ & $16.31 \pm 0.11$ & $872.9 \pm 6.2$ & $6.68 \pm 0.11$ \\
\hline 18764 & $26.74 \pm 0.12$ & $27.12 \pm 0.10$ & $18.25 \pm 0.13$ & $1089.5 \pm 8.4$ & $6.73 \pm 0.12$ \\
\hline 18765 & $25.52 \pm 0.10$ & $25.58 \pm 0.18$ & $17.26 \pm 0.12$ & $971.7 \pm 7.8$ & $6.77 \pm 0.16$ \\
\hline 18766 & $23.48 \pm 0.10$ & $23.85 \pm 0.10$ & $15.43 \pm 0.11$ & $810.2 \pm 6.0$ & $6.24 \pm 0.10$ \\
\hline 19485 & $14.89 \pm 0.13$ & $14.80 \pm 0.22$ & $8.60 \pm 0.13$ & $280.3 \pm 4.9$ & $5.26 \pm 0.17$ \\
\hline 19492 & $17.87 \pm 0.14$ & $17.71 \pm 0.15$ & $10.90 \pm 0.14$ & $424.8 \pm 5.9$ & $5.72 \pm 0.15$ \\
\hline 19494 & $20.08 \pm 0.10$ & $20.18 \pm 0.14$ & $12.63 \pm 0.11$ & $561.1 \pm 5.3$ & $5.88 \pm 0.12$ \\
\hline 19498 & $21.82 \pm 0.17$ & $21.65 \pm 0.19$ & $14.14 \pm 0.18$ & $673.6 \pm 9.3$ & $6.34 \pm 0.20$ \\
\hline 19500 & $21.84 \pm 0.10$ & $21.72 \pm 0.10$ & $14.14 \pm 0.11$ & $676.0 \pm 5.4$ & $6.31 \pm 0.11$ \\
\hline 19503 & $16.24 \pm 0.10$ & $16.34 \pm 0.10$ & $9.58 \pm 0.10$ & $344.7 \pm 3.7$ & $5.32 \pm 0.10$ \\
\hline
\end{tabular}



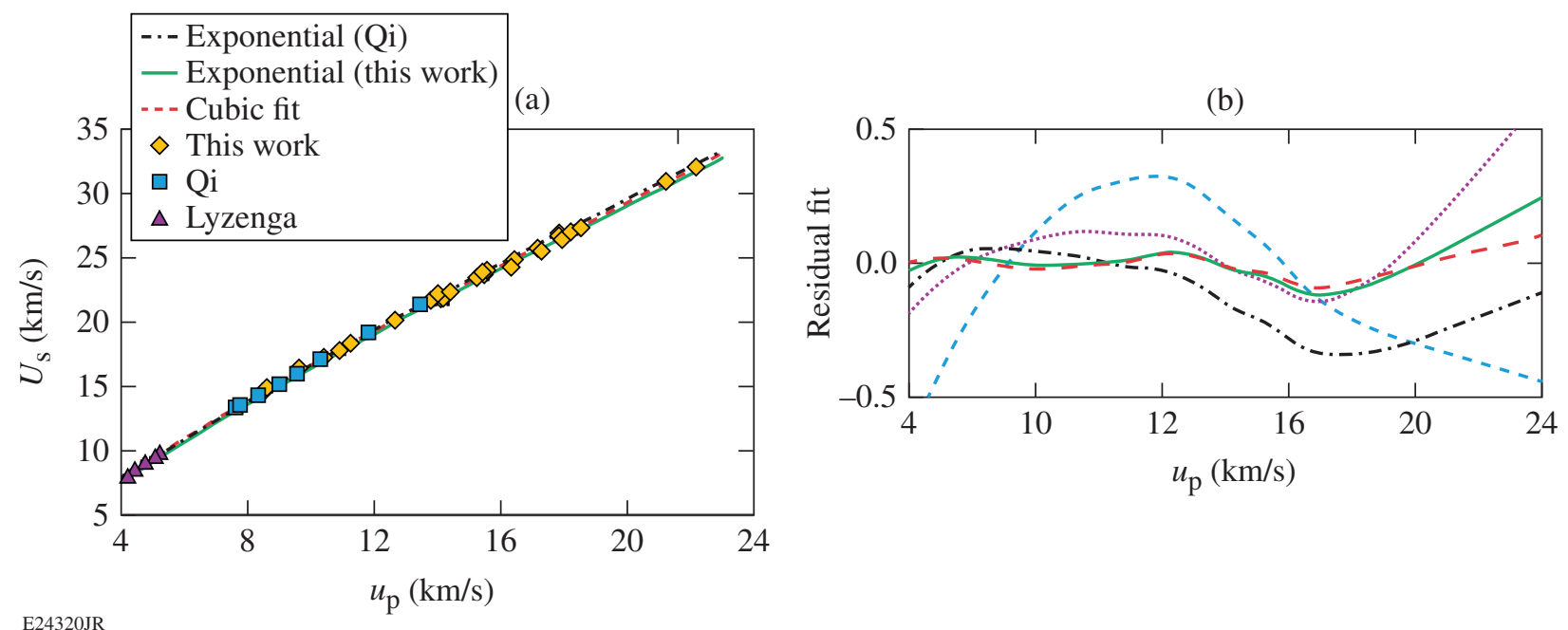

Figure 143.20

(a) $U_{\mathrm{s}}-u_{\mathrm{p}}$ plot for fused silica. Experimental results from this work (yellow diamonds), Qi et al..$^{19}$ (blue squares), and Lyzenga et al. ${ }^{7}$ (purple triangles) are shown. The exponential fit from Qi et al. (black dashed-dotted line) and cubic (red dashed line) and exponential (solid green line) fits from this work are also displayed. The error bars on the data are smaller than the points in the graph. (b) Fits to residual of $U_{\mathrm{s}}-u_{\mathrm{p}}$ relations using a low-order smoothing spline. Cubic (red dashed line) and exponential (green solid line) fits from this work agree with the data. Linear (blue dashed line) and quadratic (purple dotted line) demonstrate systematic error in the fit, and exponential fit from Qi et al. (black dashed-dotted line) diverges from experimental results for $u_{\mathrm{p}}>12 \mathrm{~km} / \mathrm{s}(P>\sim 500 \mathrm{GPa}$ ).

weighted nonlinear-least-squares methods to the data in this article and that from both Qi et al. ${ }^{19}$ and Lyzenga et al. ${ }^{7}$ The coefficients and covariance matrix for the cubic fit are given in Tables 143.II and 143.III. Similarly, the parameters for the exponential model are given in Tables 143.IV and 143.V.

Table 143.II: Fit parameters for the cubic $U_{\mathrm{s}}-u_{\mathrm{p}}$ relation of the

\begin{tabular}{|c|c|c|c|}
\multicolumn{4}{|c|}{ form $U_{\mathrm{s}}=a_{0}+a_{1} u_{\mathrm{p}}+a_{2} u_{\mathrm{p}}^{2}+a_{3} u_{\mathrm{p}}^{3}$} \\
\hline \hline$a_{0}(\mathrm{~km} / \mathrm{s})$ & $a_{1}$ & $a_{2}(\mathrm{~km} / \mathrm{s})^{-1}$ & $a_{3}(\mathrm{~km} / \mathrm{s})^{-2}$ \\
\hline 0.635 & 1.937 & $-4.102 \times 10^{-2}$ & $7.925 \times 10^{-4}$ \\
\hline
\end{tabular}

The residuals for each of these fits were computed and, for clarity, a low-order spline was fit to each set of residuals and displayed in Fig 143.20(b). Linear (blue dashed line) and quadratic (purple dotted line) fits are also shown for contrast. These residual plots show that the cubic fit and our new exponential fits replicate residual data to similar precision. Importantly, the exponential fit given by Qi et al. does not fit these data well, predicting stiffer behavior at higher pressures. This is shown in the $P-\rho$ Hugoniot data in Fig. 143.21. It should be noted that the Qi fit is based on their molecular dynamics simulations, which agreed with their data up to $\sim 630 \mathrm{GPa}$ (the highest pressure reached in their experiments). The simulations were performed for pressures from 86 to $1500 \mathrm{GPa}$ and those simulation results were the basis for their exponential fit.

Note that both our data and the fits (cubic and exponential) to our data agree very well with the data from Qi et al. It is the extrapolation of the first-principles molecular dynamics (FPMD)-based exponential fit of Qi et al. that is inconsistent with the data presented here. This can be seen in the residuals in Fig. 143.20(b), where the Qi model (black dashed-dotted line) deviates from the data at $u_{\mathrm{p}}>\sim 12(\mathrm{~km} / \mathrm{s})$. Below that velocity the models and data agree well. That velocity corresponds to $\sim 600 \mathrm{GPa}$, which is approximately the pressure determined by Hicks et al. for the transition from a bonded liquid to an atomic fluid along the Hugoniot of fused silica. ${ }^{16}$ This implies that the compressibility of the bonded liquid differs from that of the atomic fluid, with the dissociated atomic fluid being more compressible.

Table 143.III: Covariance matrix elements for the cubic $U_{\mathrm{s}}-u_{\mathrm{p}}$ relation given in Table 143.II.

\begin{tabular}{|c|c|c|c|c|c|c|c|c|c|}
\hline $\begin{array}{c}\sigma_{a_{0}}^{2} \\
\left(\times 10^{-1}\right)\end{array}$ & $\begin{array}{c}\sigma_{a_{0}} \sigma_{a_{1}} \\
\left(\times 10^{-2}\right)\end{array}$ & $\begin{array}{c}\sigma_{a_{0}} \sigma_{a_{2}} \\
\left(\times 10^{-3}\right)\end{array}$ & $\begin{array}{c}\sigma_{a_{0}} \sigma_{a_{3}} \\
\left(\times 10^{-4}\right)\end{array}$ & $\begin{array}{c}\sigma_{a_{1}}^{2} \\
\left(\times 10^{-3}\right)\end{array}$ & $\begin{array}{c}\sigma_{a_{1}} \sigma_{a_{2}} \\
\left(\times 10^{-4}\right)\end{array}$ & $\begin{array}{c}\sigma_{a_{1}} \sigma_{a_{3}} \\
\left(\times 10^{-5}\right)\end{array}$ & $\begin{array}{c}\sigma_{a_{2}}^{2} \\
\left(\times 10^{-5}\right)\end{array}$ & $\begin{array}{c}\sigma_{a_{2}} \sigma_{a_{3}} \\
\left(\times 10^{-6}\right)\end{array}$ & $\begin{array}{c}\sigma_{a_{3}}^{2} \\
\left(\times 10^{-8}\right)\end{array}$ \\
\hline 1.118 & -3.019 & 2.419 & -5.930 & 8.568 & -7.094 & 1.781 & 6.033 & -1.547 & 4.041 \\
\hline
\end{tabular}


Table 143. IV: Fit parameters for the exponential $U_{\mathrm{s}}-u_{\mathrm{p}}$ relation of the form $U_{\mathrm{s}}=a+b u_{\mathrm{p}}-c u_{\mathrm{p}} e^{-d u_{\mathrm{p}}}$.

\begin{tabular}{|c|c|c|c|}
\hline$a(\mathrm{~km} / \mathrm{s})$ & $b$ & $c$ & $d(\mathrm{~km} / \mathrm{s})^{-1}$ \\
\hline 4.972 & 1.218 & 2.432 & 0.396 \\
\hline
\end{tabular}

Table 143.V: Covariance matrix elements for the exponential $U_{\mathrm{s}}-u_{\mathrm{p}}$ relation given in Table 143.IV.

\begin{tabular}{|c|c|c|c|c|c|c|c|c|c|}
\hline $\begin{array}{c}\sigma_{a}^{2} \\
\left(\times 10^{-1}\right)\end{array}$ & $\begin{array}{c}\sigma_{a} \sigma_{b} \\
\left(\times 10^{-2}\right)\end{array}$ & $\begin{array}{c}\sigma_{a} \sigma_{c} \\
\left(\times 10^{-2}\right)\end{array}$ & $\begin{array}{c}\sigma_{a} \sigma_{d} \\
\left(\times 10^{-2}\right)\end{array}$ & $\begin{array}{c}\sigma_{b}^{2} \\
\left(\times 10^{-4}\right)\end{array}$ & $\begin{array}{c}\sigma_{b} \sigma_{c} \\
\left(\times 10^{-3}\right)\end{array}$ & $\begin{array}{c}\sigma_{b} \sigma_{d} \\
\left(\times 10^{-3}\right)\end{array}$ & $\begin{array}{c}\sigma_{c}^{2} \\
\left(\times 10^{-1}\right)\end{array}$ & $\begin{array}{c}\sigma_{c} \sigma_{d} \\
\left(\times 10^{-2}\right)\end{array}$ & $\begin{array}{c}\sigma_{d}^{2} \\
\left(\times 10^{-3}\right)\end{array}$ \\
\hline 1.900 & -1.005 & -4.749 & -2.101 & 5.386 & 2.116 & 1.075 & 1.004 & 1.149 & 2.809 \\
\hline
\end{tabular}

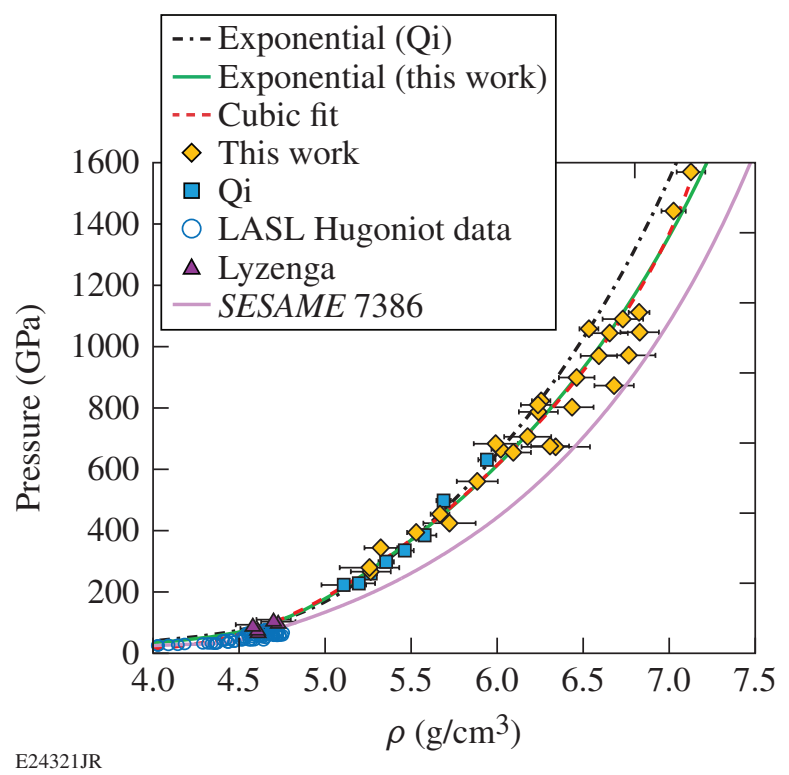

Figure 143.21

$P-\rho$ Hugoniot curve. The exponential fit from Qi et al. ${ }^{19}$ clearly lies above the data and fits computed using this work and data from Qi $e t$ al. and Lyzenga et al. ${ }^{7}$ This implies that the fit by Qi is less compressible than the experimental data. Exponential fit and cubic fit from this work are indistinguishable for pressures $<1400 \mathrm{GPa}$. Above $1400 \mathrm{GPa}$, the cubic fit begins to diverge and is not valid for extrapolation to pressures $>1600 \mathrm{GPa}$. All data use the same labels as Fig. 143.20(a). Los Alamos Scientific Laboratory shock Hugoniot data (open blue circles) and the SESAME 7386 table (magenta line) are included for completeness.

\section{Conclusion}

The Hugoniot of fused silica shocked to the fluid state was measured by impedance matching to $\alpha$-quartz for pressures up to $1600 \mathrm{GPa}$. The results from these laser-driven experiments are in good agreement over the range of 200 to $600 \mathrm{GPa}$ with results using magnetically driven aluminum flyer plates, a significant departure from past experience comparing the two drive platforms. The results extend the measurements for the fused- silica Hugoniot well into the region where silica is expected to dissociate into an atomic fluid. This may explain why recent FPMD predictions by Qi et al. ${ }^{22}$ underpredict the compressibility of silica at the higher pressures. Using this data and that of Qi and Lyzenga, a new $U_{\mathrm{s}}-u_{\mathrm{p}}$ curve was generated for the range of 200 to $1600 \mathrm{GPa}$. The cubic polynomial presented in Table 143.II and a fit using the form $U_{\mathrm{s}}=a+b u_{\mathrm{p}}-c u_{\mathrm{p}} e^{-d u_{\mathrm{p}}}$ given in Table 143.IV were found to model the data equally well. The significant curvature in the cubic model is undesirable for extrapolation to pressures above $1600 \mathrm{GPa}$. Therefore, the exponential model is preferred for extrapolation since at higher pressures it asymptotes to a linear $U_{\mathrm{s}}-u_{\mathrm{p}}$ relationship.

\section{ACKNOWLEDGMENT}

This material is based upon work supported by the Department of Energy National Nuclear Security Administration under Award Number DE-NA0001944, the University of Rochester, and the New York State Energy Research and Development Authority. The support of DOE does not constitute an endorsement by DOE of the views expressed in this article.

\section{REFERENCES}

1. J. W. Morgan and E. Anders, Proc. Natl. Acad. Sci. 77, 6973 (1980).

2. S. K. Saxena, Geochim. Cosmochim. Acta 60, 2379 (1996).

3. Y. Fei, H.-K. Mao, and B. O. Mysen, J. Geophys. Res. 96, 2157 (1991).

4. R. J. Hemley, C. T. Prewitt, and K. J. Kingma, Rev. Mineral. Geochem. 29, 41 (1994).

5. L. S. Dubrovinsky et al., Nature 388, 362 (1997).

6. D. Andrault et al., Science 282, 720 (1998).

7. Q. Williams and R. Jeanloz, Science 239, 902 (1988).

8. J. Wackerle, J. Appl. Phys. 33, 922 (1962).

9. R. G. McQueen, J. N. Fritz, and S. P. Marsh, J. Geophys. Res. 68, 2319 (1963). 
10. G. A. Lyzenga, T. J. Ahrens, and A. C. Mitchell, J. Geophys. Res. B 88, 2431 (1983).

11. S.-N. Luo et al., Phys.-Usp. 45, 435 (2002).

12. S.-N. Luo et al., Geophys. Res. Lett. 29, 36 (2002).

13. J. A. Akins and T. J. Ahrens, Geophys. Res. Lett. 29, 31 (2002).

14. D. G. Hicks, T. R. Boehly, P. M. Celliers, J. H. Eggert, E. Vianello, D. D. Meyerhofer, and G. W. Collins, Phys. Plasmas 12, 082702 (2005).

15. M. D. Knudson and M. P. Desjarlais, Phys. Rev. Lett. 103, 225501 (2009).

16. D. G. Hicks, T. R. Boehly, J. H. Eggert, J. E. Miller, P. M. Celliers, and G. W. Collins, Phys. Rev. Lett. 97, 025502 (2006).

17. T. R. Boehly, J. E. Miller, D. D. Meyerhofer, J. G. Eggert, P. M. Celliers, D. G. Hicks, and G. W. Collins, in Shock Compression of Conducted Matter-2007, edited by M. Elert, M. D. Furnish, R. Chau, N. Holmes, and J. Nguyen (American Institute of Physics, Melville, NY, 2007), Vol. 955, pp. 19-22.

18. S. Brygoo, D. G. Hicks, P. Loubeyre, J. H. Eggert, S. McWilliams, P. M. Celliers, T. R. Boehly, R. Jeanloz, and G. W. Collins, "Development of Melted Quartz as an Impedance-Matching Standard for Strong Laser Shock Measurements (unpublished)."

19. M. D. Knudson and M. P. Desjarlais, Phys. Rev. B 88, 184107 (2013).

20. S. Brygoo et al., Nature Materials 6, 274 (2007).

21. M. Millot et al., Science 347, 418 (2015).

22. T. Qi et al., Phys. Plasmas 22, 062706 (2015).

23. R. G. Kraus et al., J. Geophys. Res., E, Planets 117, E09009 (2012).

24. J. E. Miller, "High-Pressure Equation-of-State of Porous- $\mathrm{Ta}_{2} \mathrm{O}_{5}$," Ph.D. thesis, University of Rochester, 2007.
25. M. D. Knudson and R. W. Lemke, J. Appl. Phys. 114, 053510 (2013).

26. K. Falk, C. A. McCoy, C. L. Fryer, C. W. Greeff, A. L. Hungerford, D. S. Montgomery, D. W. Schmidt, D. G. Sheppard, J. R. Williams, T. R. Boehly, and J. F. Benage, Phys. Rev. E 90, 033107 (2014).

27. T. Lay, Q. Williams, and E. J. Garnero, Nature 392, 461 (1998).

28. M. B. Boslough and J. R. Asay, in High-Pressure Shock Compression of Solids, edited by J. R. Asay and M. Shahinpoor, High-Pressure Shock Compression of Condensed Matter (Springer, New York, 1993), pp. 7-42.

29. T. R. Boehly, Laboraty for Laser Energetics, private communication (2014).

30. L. J. Waxer, D. N. Maywar, J. H. Kelly, T. J. Kessler, B. E. Kruschwitz, S. J. Loucks, R. L. McCrory, D. D. Meyerhofer, S. F. B. Morse, C. Stoeckl, and J. D. Zuegel, Opt. Photonics News 16, 30 (2005).

31. Y. Lin, T. J. Kessler, and G. N. Lawrence, Opt. Lett. 20, 764 (1995).

32. M. A. Barrios, D. G. Hicks, T. R. Boehly, D. E. Fratanduono, J. H. Eggert, P. M. Celliers, G. W. Collins, and D. D. Meyerhofer, Phys. Plasmas 17, 056307 (2010).

33. B. L. Henke, E. M. Gullikson, and J. C. Davis, At. Data Nucl. Data Tables 54, 181 (1993).

34. L. M. Barker and R. E. Hollenbach, J. Appl. Phys. 43, 4669 (1972).

35. L. M. Barker and K. W. Schuler, J. Appl. Phys. 45, 3692 (1974).

36. P. M. Celliers et al., Appl. Phys. Lett. 73, 1320 (1998).

37. P. M. Celliers, D. K. Bradley, G. W. Collins, D. G. Hicks, T. R. Boehly, and W. J. Armstrong, Rev. Sci. Instrum. 75, 4916 (2004).

38. M. A. Barrios, T. R. Boehly, D. G. Hicks, D. E. Fratanduono, J. H. Eggert, G. W. Collins, and D. D. Meyerhofer, J. Appl. Phys. 111, 093515 (2012). 